\title{
Expectation values, Lorentz invariance, and CPT in the open bosonic string
}

\author{
V. Alan Kostelecký ${ }^{a}$, R. Potting ${ }^{b}$ \\ a Physics Department, Indiana University, Bloomington, IN 4740S, USA \\ ${ }^{\text {h }}$ U.C.E.H., Universidade do Algarve, Campus de Gambelas, 8000 Faro, Portugal
}

Received 2 November 1995; revised manuscript received 14 November 1995

Editor: H. Georgi

\begin{abstract}
The issue of spontaneous breaking of Lorentz and CPT invariance is studied in the open bosonic string using a truncation scheme to saturate the string-field action at successively higher levels. We find strong evidence for the existence of extrema of the action with nonzero expectation values for certain fields. The Lorentz- and CPT-preserving solution previously suggested in the literature is confirmed through level 12 in the action. A family of Lorentz-breaking, CPT-preserving solutions of the equations of motion is found to persist and converge through level 18 in the action. Two sequences of solutions spontaneously breaking both Lorentz invariance and CPT are discussed. The analysis at this level involves the analytical form of over 20,000 terms in the static potential.
\end{abstract}

\section{Introduction}

Many string theories in their critical dimension suffer from instability due to scalar tachyons in their spectra. It is natural to suspect that these theories could be stabilized in a different vacuum determined by nonzero expectation values for some fields. A relatively simple example is the open bosonic string, for which the existence of a covariant string field theory [1] makes it possible in principle to seek solutions of the field equations that exhibit nonzero expectation values for the tachyon and perhaps other fields. Using a truncation scheme based on the Mandelstam notion [2] of amplitude saturation, evidence has been found for the existence of a nontrivial solution in which stability is restored and modifications to the physics arise [3].

When the critical dimension exceeds four, the higher-dimensional Lorentz invariance must also be broken to produce a physically realistic model. A natural stringy mechanism has been identified that can induce spontaneous Lorentz-symmetry breaking [4], an effect that is impossible in conventional renormalizable particle gauge theories. Under suitable circumstances, spontaneous CPT breaking can then also occur [5]. However, to date no explicit solutions of covariant string field theory have been presented that display spontaneous Lorentz (and CPT) breaking. In this letter, we address this gap in the literature.

\section{Methodology}

In the covariant field theory for the open bosonic string, Fourier decomposition of the string coordinates allows an expansion of the string field $\Phi$ as a linear combination of particle fields dependent on the string 
center-of-mass coordinate $x_{0}^{\mu}[6]$. In terms of real particle fields and in the Siegel-Feynman gauge [7], the expansion begins: ${ }^{1}$

$$
\begin{gathered}
|\Phi\rangle=\left[\phi\left(x_{0}\right)+A_{\mu}\left(x_{0}\right) \alpha_{-1}^{\mu}+\frac{1}{\sqrt{2}} i B_{\mu}\left(x_{0}\right) \alpha_{-2}^{\mu}\right. \\
+\frac{1}{\sqrt{2}} B_{\mu \nu}\left(x_{0}\right) \alpha_{-1}^{\mu} \alpha_{-1}^{\nu}+\beta_{1}\left(x_{0}\right) b_{-1} c_{-1}+\ldots \\
\left.+\frac{1}{2} i D_{\mu}\left(x_{0}\right) \alpha_{-4}^{\mu}+\ldots+\delta_{3}\left(x_{0}\right) b_{-1} c_{-3}+\ldots\right]\left|-\frac{1}{2}\right\rangle .
\end{gathered}
$$

In the usual vacuum, $\phi$ is the tachyon, $A_{\mu}$ is the massless vector, $B_{\mu}$ and $B_{\mu \nu}$ are fields of squared mass one, $D_{\mu}$ is a field of squared mass three, and the remaining fields are auxiliary. The first-quantized string vacuum is denoted $\left|-\frac{1}{2}\right\rangle$. The various combinations of the creation operators $\alpha_{-n}^{\mu}, b_{-n}, c_{-n}$ acting on the vacuum span a basis in the string Fock space. The normalization factors of $1 / \sqrt{2}$ and $1 / 2$ are chosen to ensure canonical kinetic terms in the string field action in the conventions of Refs. [3,5]. By definition, the level of a field is its mass squared in units of $1 / \alpha^{\prime}$ as measured from the tachyon level. Thus, the tachyon is at level zero, while $A_{\mu}$ is at level one and the $B$ fields are at level two.

Using this expansion, the string field theory can be expressed as a particle field action involving an infinite number of fields and an infinite number of cubic couplings. We are interested in nontrivial (stable or unstable) vacua of the full action, i.e. solutions to the equations of motion. Since an exact analysis remains impractical at present, we base our analysis on a leveltruncation scheme in which the string field expansion (1) is truncated at successively higher levels. For clarity in what follows, we say that a trunction is at level $(m, n)$ when all fields up to and including level $m$ appear in the field expansion and all terms up to and including total level $n$ are taken in the cubic interactions. Here, the total level number of a given term in the action is defined as the sum of the level numbers of the fields in that term. Further details about the leveltruncation scheme and a test in a situation where the exact answer is known may be found in Ref. [3].

\footnotetext{
I Throughout this work except where stated, we adopt the conventions of Refs. [3,5] with the choice $g=\alpha^{\prime}=1$.
}

We seek static solutions of the equations of motion, so only the static part of the cubic potential is needed. Even with this restriction, the length of the action grows very rapidly as the truncation level is increased. Under certain circumstances where all odd-level fields have vanishing expectation values, a further simplification can be made by excluding odd-level fields $a$ priori. Other possible simplifications include considering only expectation values of Lorentz scalars, or allowing only expectation values along one direction in spacetime. Nonetheless, the fierce growth of the number of terms in the action with level places practical constraints on the truncation depth to which calculations are feasible.

We use symbolic-manipulation software to produce the analytical form of the action at a specified truncation level and to derive the corresponding equations of motion. These are simultaneous coupled polynomial equations, one for each field appearing at the chosen truncation level. An iterative procedure is then applied to these equations to obtain their roots, each of which is a set of ficld-cxpectation values. For low truncation levels it is possible to find all the roots, but typically it is necessary to perform a search to obtain extrema of interest.

Since we are interested in solutions that persist at arbitrarily high truncations, it is useful to have some criteria to dismiss unpromising candidate roots. For instance, vacua with nonzero imaginary parts for any field are excluded. We also discard roots for which the expectation value of any field or the value of the action in the new vacuum is significantly greater than the truncation level. As an additional check on an otherwise acceptable solution, we performed the following test, referred to below as the $x$ test. It is based on the idea that at any given truncation level $(m, n)$ a satisfactory solution should be dominated by expectation values of lower-level fields. We therefore take the relevant action at level $(m, n)$, multiply each term at total level number $k$ by $x^{k}$, and substitute the root to be tested. The result is a polynomial in the dummy variable $x$, with the numerical coefficient of the $k$ th power of $x$ determining the relative contribution to the action of the couplings at level $k$ for the given root. A good solution is expected to display coefficients falling rapidly with $k$. Also, to avoid accidental misidentification of roots, where feasible at each truncation level we have followed the evolution of each root as the co- 
efficients of higher-truncation terms in the action are gradually increased from zero to their full value. The sequences of solutions we present below are adiabatically connected in this sense.

For brevity in what follows, we present the behavior of only those fields shown in Eq. (1). Also, it is calculationally convenient to disregard the normalization factors of $1 / \sqrt{2}$ and $1 / 2$ appearing in Eq. (1), so the numerical expectation values presented below must be appropriately scaled if they are to correspond to canonically normalized fields in our conventions.

\section{Lorentz-invariant solution}

In the present notation, the truncation sequence for the Lorentz-invariant solution of Ref. [3] is $(0,0)$, $(2,4),(2,6)$. At level $(2,6)$, the nontrivial expectation values in this sequence involve the fields $\phi, \beta_{1}$, and ${ }^{2} B=\eta^{\mu \nu} B_{\mu \nu} / 26$. At this truncation level, the expectation values of these fields and the resulting action $S$ when expressed in our dimensionless units are ${ }^{3}$

$\phi=1.088, \quad \beta_{1}=0.3804, \quad B=0.05596$, $S=-0.1944$.

We have determined the actions for the additional truncations $(4,4),(4,8)$, and $(4,12)$. The most stringent test of convergence is provided by the latter, which involves 13 fields with 258 terms in the cubic part of the action. Wc find excellent agreement between the results of the $(2,6)$ and $(4,12)$ truncations. The expectation value of the tachyon changes by less than one percent, and the action changes by about three percent. The largest change, of about eight percent, is in the auxiliary field $\beta_{1}$. The percentage changes relative to the $(4,8)$ truncation are much smaller than these.

The new expectation values for fields up to level two at truncation $(4,12)$ are

\footnotetext{
${ }^{2}$ Note that the normalization of $B$ wc use in this section differs by a factor $\sqrt{52}$ from that in Ref. [3].

${ }^{3}$ This solution is close to the canonical unstable vacuum in the sense that the magnitudes of field-expectation values exhibit a rapid decrease with the corresponding masses. It and the other solutions discussed below are unlikely to be among the large class of exact solutions generated in Refs. [8] at the level of string field theory from the unnormalizable state created by the identity functional.
}

$\phi=1.097, \quad \beta_{1}=0.4113, \quad B=0.05692$,

$S=-0.2002$.

Several fields at the fourth level also acquire expectation values. In particular, the field $\delta_{3}$ in Eq. (1) acquires the value $\delta_{3}=0.1124$. As expected for a convergent solution, the largest values at level four are clearly smaller than the largest ones at level two. The $x$ test is well satisfied.

Fig. 1 displays the values of the field expectations and the action as a function of maximum total level of the cubic couplings. Convergence of the sequence is apparent. These results provide strong evidence for a nontrivial Lorentz-invariant solution in the open bosonic string.

\section{Lorentz-breaking, CPT-invariant solutions}

The general situation with Lorentz breaking is complicated, since the vacuum value of each component of each tensor could in principle have its own magnitude. We restricted our calculations to those special cases for which all nontrivial tensor vacuum values are diagonal and have equal-magnitude nonzero values for only a fixed subset of $d$ of the 26 possible index choices. For brevity, we focus our attention here on the special case $d=1$, although in fact we have obtained the analogues for the cases of arbitrary $d \neq 1$ of the $d=1$ sequence of Lorentz-breaking, CPT-preserving vacua presented in this section. ${ }^{4}$

For $d=1$, any Lorentz tensor appearing in the action can be replaced with a single quantity representing the possible expectation value. We found it practicable to obtain the action with all fields (independent of CPT properties) to truncation level $(6,18)$, where there are 20,620 independent cubic couplings after the simplifications from the elimination of Lorentz indices are performed. ${ }^{5}$ The remainder of this section specifically deals with the CPT-preserving case.

\footnotetext{
${ }^{4}$ Note that the existence of one nontrivial Lorentz-breaking vacuum implies the existence of others. Assuming for simplicity the euclidean formulation of the theory in which the Lorentz symmetry is the group $O(26)$, there is a $d(26-d)$-parameter family of transformations converting any given root into other Lorentzbreaking solutions.

${ }^{5}$ The reader interested in the details of these cubic couplings can find their analytical form on the World Wide Web at http://physics.indiana.edu/ kostelec/sft.html.
} 


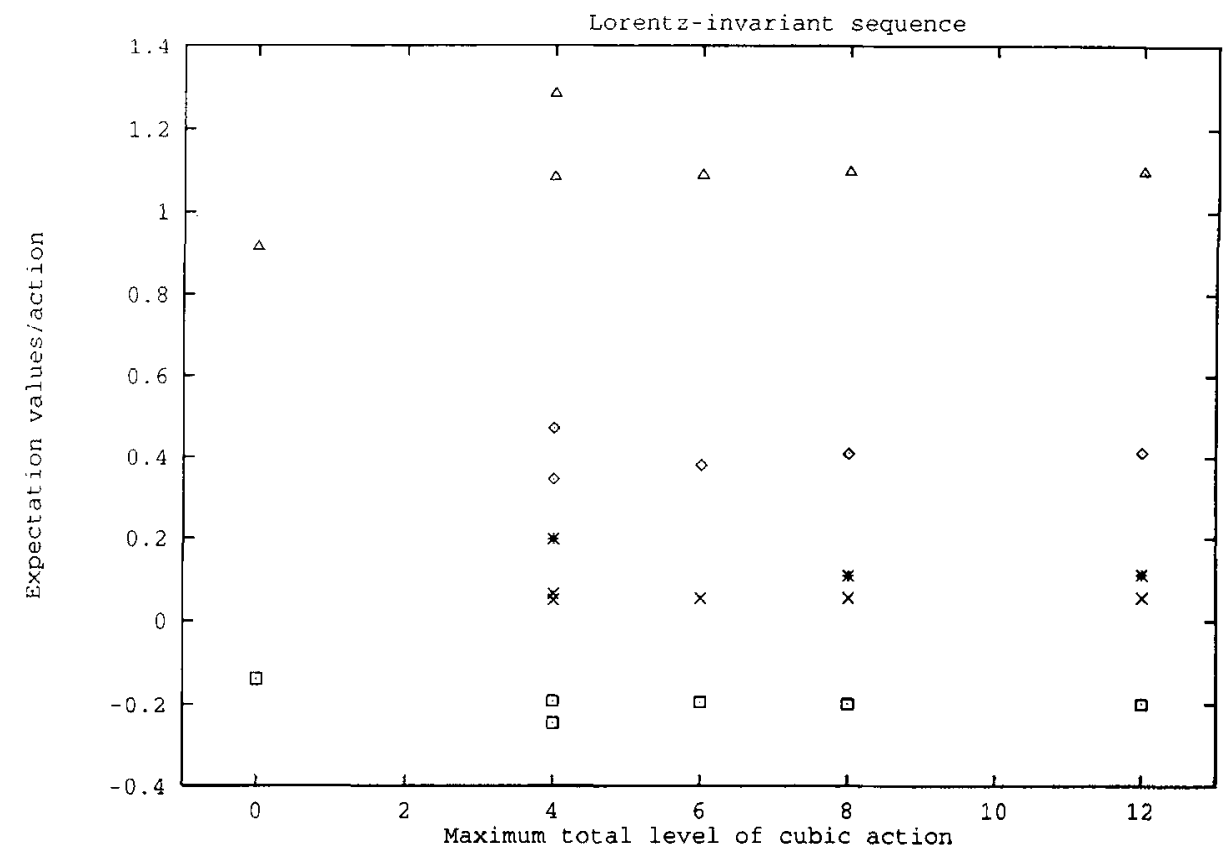

Fig. 1. Expectation values and action for the Lorentz-invariant sequence. Shown are the expectation values of the fields $\phi$ (triangles), $\beta_{1}$ (diamonds), $B$ (crosses), $\delta_{3}$ (stars) and the action (squares) at different values of the maximum total level number of the cubic interactions in the static potential of the open bosonic string.

At truncation level $(2,6)$, a complete list of solutions reveals only one nontrivial Lorentz-breaking and CPT-preserving case satisfying the criteria described in Section 2. It is

$\phi=0.7131, \quad \beta_{1}=0.2546, \quad B=0.03979$,

$S=-0.09502$.

Here, the field $B$ is taken to be the nonvanishing component of $B_{\mu \nu}$. This solution is part of a sequence that we have followed from level $(0,0)$ (identical for all $d$ to the 26-dimensional solution given in Section 3 ) through levels $(2,2),(4,4),(2,6),(6,6),(8,8)$, $(6,12)$ and $(6,18)$.

Fig. 2 shows selected field expectation values and the action as a function of maximum total level of the cubic couplings. Two truncations at total level six are displayed, namely, $(2,6)$ and $(6,6)$. It is evident that the sequence is converging. Both the subsequences $(0,0),(2,6),(4,12),(6,18)$ and $(0,0)$, $(2,2),(4,4),(6,6),(8,8)$ also appear to converge to the same value.

The vacuum values of the representative fields for the solution at truncation $(6,18)$ are: $\phi=0.6549, \quad \beta_{1}=0.2506, \quad B=0.03839$,

$S=-0.08439$.

The representative level-four field acquires an expectation $\delta_{3}=0.07081$. The $x$ test applied to this solution gives a convincing demonstration of rapid falloff of contributions with field level. At all truncation levels, this vacuum involves nonzero expectation values for even-level fields only. The results in Eq. (5) are therefore also valid at truncation level $(7,21)$, where the contributions to the cubic couplings number in the hundreds of thousands. In any event, the sequence shown strongly suggests the persistence and convergence at arbitrarily high truncations of a nontrivial Lorentz-breaking solution in the open bosonic string.

\section{Lorentz- and CPT-breaking solutions}

Spontaneous CPT breaking requires a nonzero expectation value for a Lorentz tensor with an odd number of indices. A list of all solutions at truncation level $(2,6)$ displays the existence of two independent ac- 


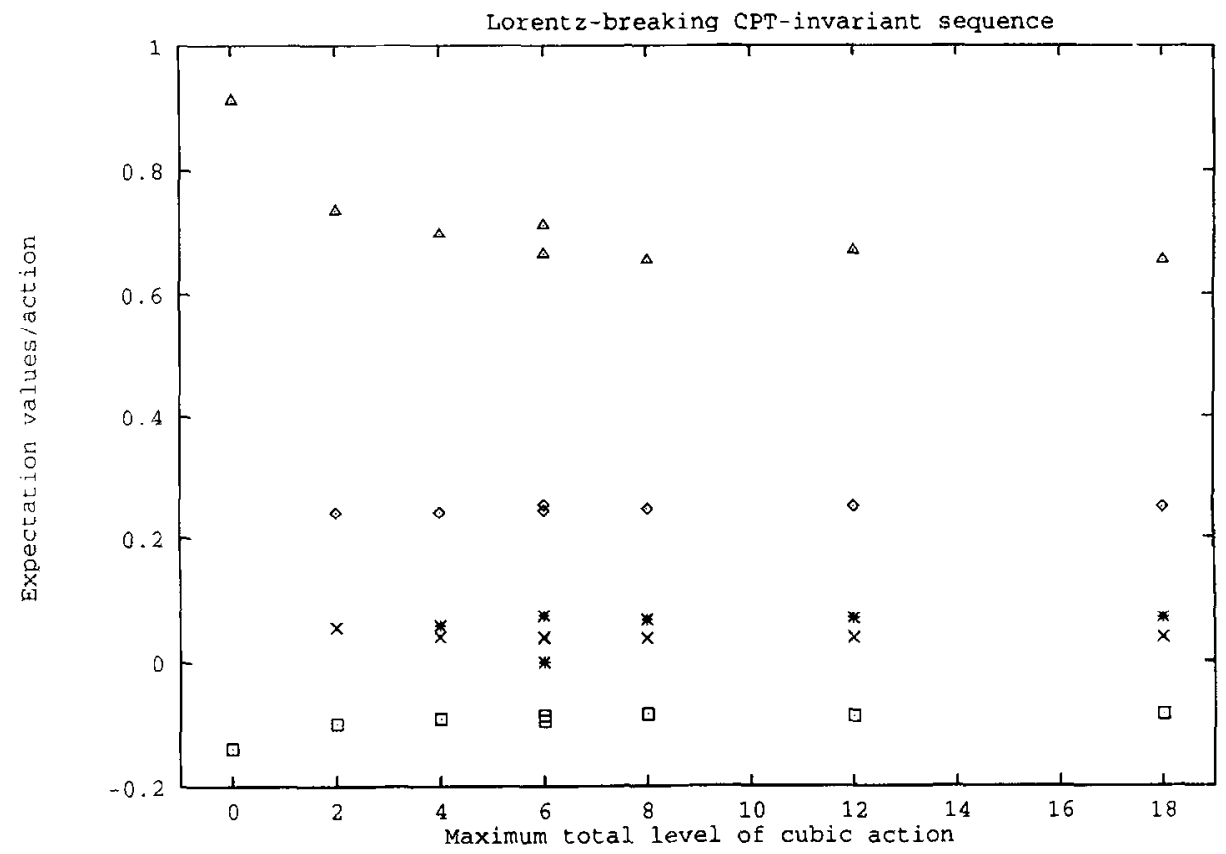

Fig. 2. Expectation values and action for the Lorentz-breaking, CPT-invariant sequence for the case $d=1$. Shown are the expectation values of the fields $\phi$ (triangles), $\beta_{1}$ (diamonds), $B$ (crosses), $\delta_{3}$ (stars) and the action (squares) at different values of the maximum total level number of the cubic interactions in the static potential of the open bosonic string.

ceptable CPT-breaking roots, plus their CPT images. One is

$\phi=-0.5607, \quad \beta_{1}=-0.2479, \quad B 1= \pm 1.154$,

$B 2=-0.9735, \quad S=-0.1908$.

Here, we denote by $B 1$ the nonvanishing component of the one-index field $B_{\mu}$ appearing in Eq. (1) and by $B 2$ the nonvanishing component of the two-index field $B_{\mu \nu}$. The field $B 1$ can have either sign, corresponding to the two CPT conjugate solutions. The other root at truncation level $(2,6)$ is

$\phi=-0.8192, \quad \beta_{1}=-0.4104, \quad B 1= \pm 1.431$,

$B 2=0.5735, \quad S=-0.7129$.

We have obtained roots at higher truncation levels that correspond to the above solutions. Fig. 3 shows representative expectations and the action for the first CPT-breaking sequence, involving the vacuum (6). For this first sequence five roots, at levels $(2,6)$, $(6,6),(8,8),(4,12)$, and $(6,18)$, could be confirmed to be adiabatically connected. The first two are so similar that the different values of $\phi$ and $B 2$ are indistinguishable on the scale used. The figure suggests the persistence of this first CPT-breaking sequence at high level and is consistent with its convergence to a finite solution as the truncation level is further increased. At truncation level $(6,18)$, the values of the fields up to level two and the action are:

$\phi=-1.798, \quad \beta_{1}=-1.085, \quad B 1= \pm 2.783$,

$B 2=-0.7327, \quad S=-1.348$.

The values of the representative level-four fields are $\delta_{3}=-0.2676$ and $D=0.1602$. Here, $D$ is the nonvanishing component of the level-four field $D_{\mu}$.

Fig. 4 displays the analogous quantities for the second CPT-breaking sequence, involving the root (7). In this case six adiabatically connected roots were found, involving truncation levels $(4,4),(2,6)$, $(6,6),(8,8),(4,12)$, and $(6,18)$. At truncation level $(6,18)$, the values of the fields up to level two and the action are:

$\phi=-1.892, \quad \beta_{1}=-2.407, \quad B 1= \pm 3.369$.

$B 2=1.679, \quad S=-2.865$.

The values of the representative level-four fields are $\delta_{3}=-0.09983$ and $D=-0.01003$. The figure pro- 


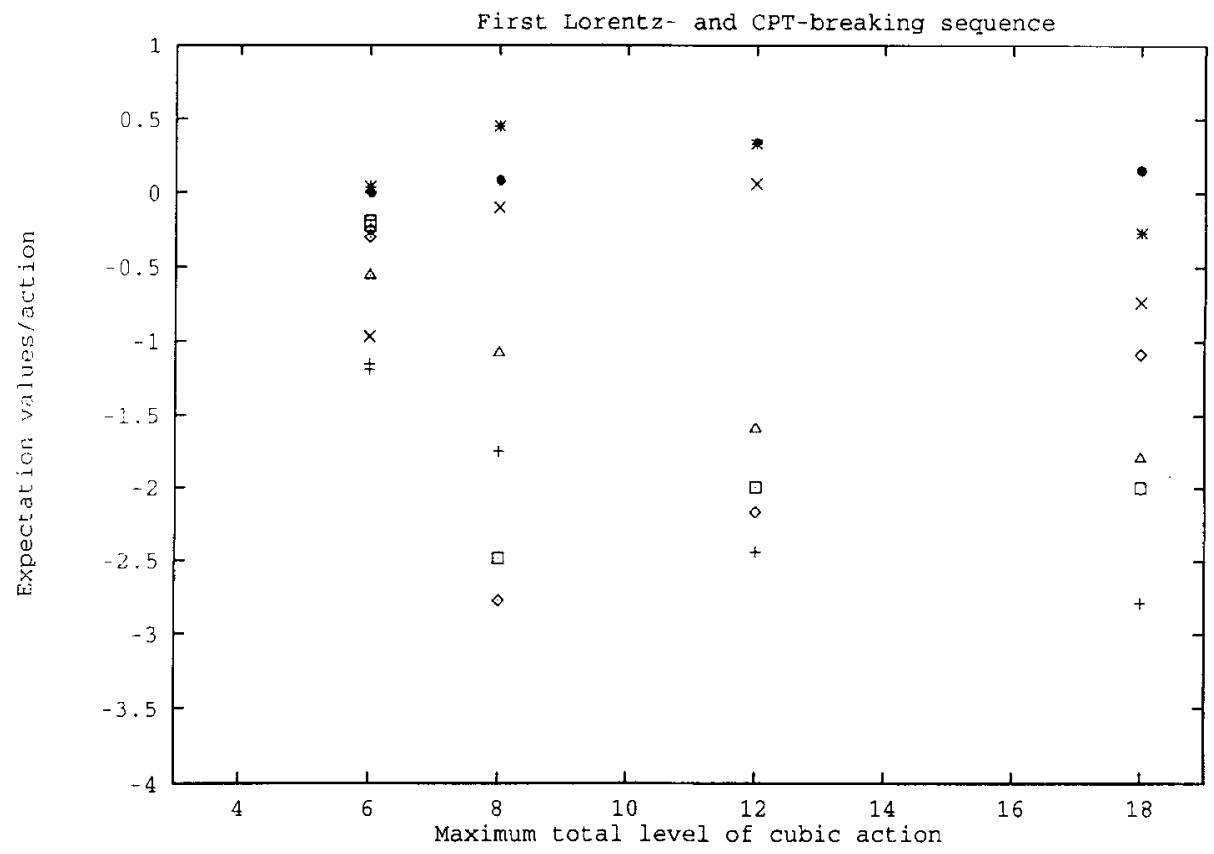

Fig. 3. Expectation values and action for the first Lorentz- and CPT-breaking sequence for the case $d=1$. Shown are the expectation values of the fields $\phi$ (triangles), $\beta_{1}$ (diamonds), $B 2$ (crosses), $B 1$ (pluses), $D$ (dots), $\delta_{3}$ (stars) and the action (squares) at different values of the maximum total level number of the cubic interactions in the static potential of the open bosonic string.

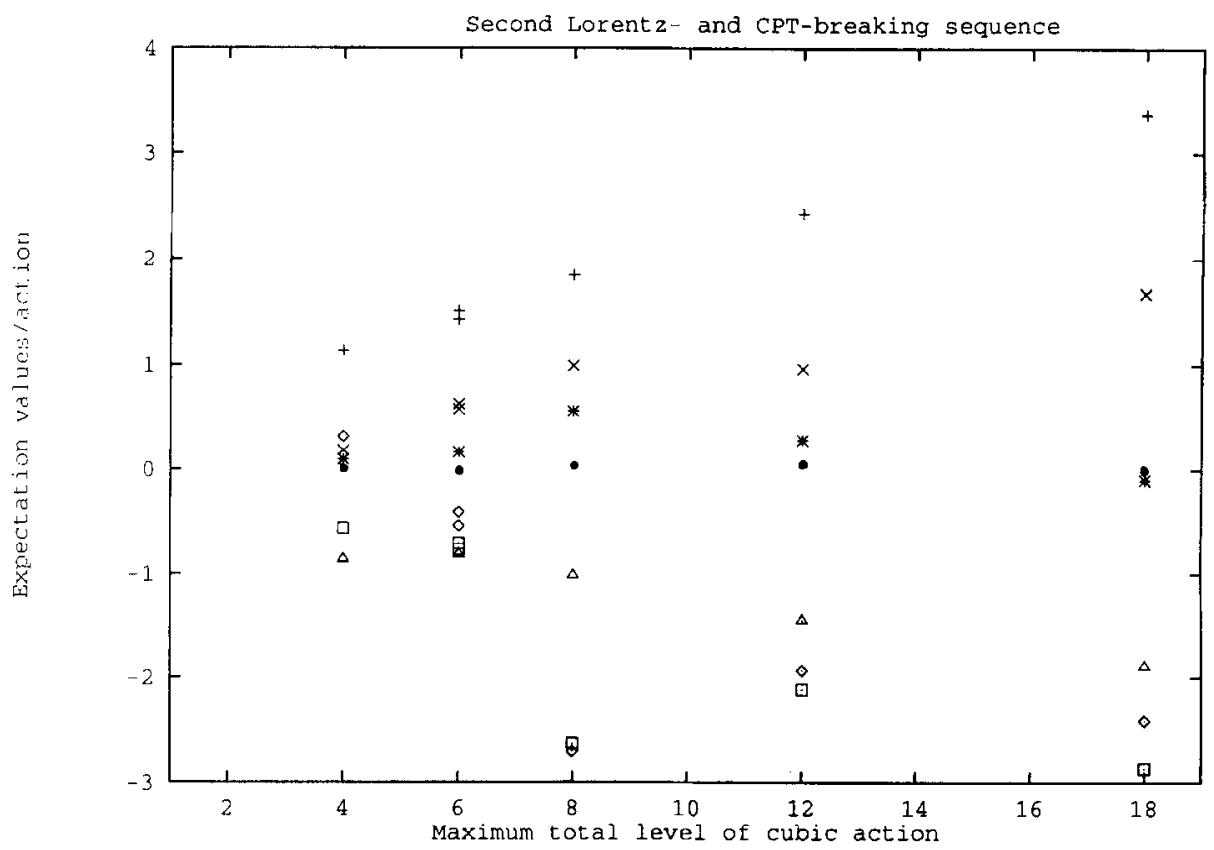

Fig. 4. Expectation values and action for the second Lorentz- and CPT-breaking sequence for the case $d=1$. Shown are the expectation vaiues of the fields $\phi$ (triangles), $\beta_{1}$ (diamonds), $B 2$ (crosses), $B 1$ (pluses), $D$ (dots), $\delta_{3}$ (stars) and the action (squares) at different values of the maximum total level number of the cubic interactions in the static potential of the open bosonic string. 
vides good support for the consistency of the second sequence. However, the expectation of the level-two CPT-breaking field $B 1$ appears to grow almost linearly with total level number. This suggests that the sequence may be unbounded in the limit of the full theory, even if the solution exists at any finite truncation level. In both cases the $x$-test polynomials do display the desired behavior of decreasing coefficients with total level number, but the fall-off is significantly less rapid than for the solution in Section 4. It seems that, if either CPT-breaking sequence eventually saturates, the rate of convergence is considerably smaller than for the CPT-preserving case.

\section{Comments}

The solutions found above all have vanishing values for odd-level fields. A priori, we know of no reason why this must be so. A separate search for CPTbreaking solutions with nonzero expectation for the level-1 vector $A_{\mu}$ yielded only one candidate at or below level $(3,9)$ that passed our preliminary cuts. However, this solution is unsatisfactory as it does not generate an acceptable sequence. It also fails the $x$ test, producing a polynomial with coefficients oscillating by several orders of magnitude in successive terms.

The nontrivial extrema we have found in the previous sections display features expected from the general analysis suggested in Refs. [4,5]. For example, the mechanism for spontaneous Lorentz breaking described in Ref. [4] relies here on the appearance of terms of the form $S T \cdot T$, where $T$ is a Lorentz tensor and $S$ is a scalar. If a scalar with linear cubic couplings of this type acquires a wrong-sign expectation, the corresponding tensor is destabilized. In the open bosonic string, the dominant scalar is the tachyon. As expected, we find that the nontrivial Lorentz-invariant solutions presented above have positive expectation values for the tachyon, while the Lorentz-breaking ones have negative expectation values.

In principle, in a physically realistic string model the spontaneous Lorentz and CPT breaking could extend to the physical four-dimensional spacetime. If so, the process must incorporate some suppression mechanism, perhaps based on a decoupling theorem if the observed feature of no CPT violation by low-level fields extends to the general case. Since CPT invari- ance is a fundamental property of particle field theories [9], a spontaneous CPT breakdown of this type could provide an experimental signature for strings $[5,10]$. This would occur within the framework of conventional quantum mechanics and is therefore distinct from the suggested possible CPT violations that might arise in quantum gravity [11].

\section{Acknowledgements}

We thank R. Bluhm and S. Samuel for useful discussions. This work was supported in part by the United States Department of Energy under grant number DE-FG02-91ER40661 and by the Junta Nacional de Investigação Científica e Tecnológica, Portugal.

\section{References}

[1] E. Witten, Nucl. Phys. B 268 (1986) 253.

[2] S. Mandelstam, Nucl. Phys. B 64 (1973) 205; B 69 (1974) 77.

[3] V.A. Kostelecký and S. Samuel, Nucl. Phys. B 336 ( 1990) 263; Phys. Rev. Lett. 64 (1990) 2238: Phys. Rev. D 42 (1990) 1289

[4] V.A. Kostelecký and S. Samuel, Phys. Rev. D 39 (1989) 683; D 40 (1989) 1886; Phys. Rev. Lett. 63 (1989) 224; 66 (1991) 1811.

[5] V.A. Kostelecký and R. Potting, Nucl. Phys. B 359 (1991) 545.

[6] E. Cremmer, A. Schwimmer and C. Thorn, Phys, Lett. B 179 (1986) 57; S. Samuel, Phys. Lett. B 181 (1986) 255;

D.J. Gross and A. Jevicki, Nucl. Phys. B 283 (1987) 1, 225; B 293 (1987) 29.

[7] W. Siegel, Phys. Lett. B 142 (1984) 276; B 151 (1985) $391,396$.

[8] G.T. Horowitz, J. Lykken, R. Rohm and A. Strominger, Phys. Rev. Lett. 57 (1986) 283;

G.T. Horowitz, J. Morrow-Jones, S.P. Martin and R.P. Woodard, Phys. Rev. Lett. 60 (1988) 261;

L.J. Romans, Nucl. Phys. B 298 (1988) 369.

[9] J. Schwinger, Phys. Rev. 82 (1951) 914;

G. Lüders, Det. Kong. Danske Videnskabernes Selskab Mat.fysiske Meddelelser 28, no. 5 (1954); Ann. Phys. (N.Y.) 2 (1957) 1 ;

J.S. Bell, Birmingham University thesis (1954); Proc. Roy. Soc. (London) A 231 (1955) 479;

W. Pauli, p. 30 in W. Pauli, Ed., Niels Bohr and the Development of Physics (McGraw-Hill, New York, 1955); G. Lüders and B. Zumino, Phys. Rev. 106 (1957) 345; R.F. Streater and A.S. Wightman, PCT, Spin, and Statistics and All That (Benjamin Cummings, Keading, 1964); 
R. Jost, The General Theory of Quantized Fields (AMS, Providence, 1965).

101 V.A. Kostelecký, R. Potting and S. Samuel, in: S. Hegarty et al., Eds., Proc. 1991 Joint Intl. Lepton-Photon Symp. and Europhysics Conf. on High Energy Physics (World Scientific, Singapore, 1992);

D.B. Cline, ed., Gamma Ray-Neutrino Cosmology and Planck Scale Physics (World Scientific, Singapore, 1993) (hep-th/9211116);

V.A. Kostelecký and R. Potting, Phys. Rev. D 51 (1995)
3923;

D. Colladay and V.A. Kostelecký, Phys. Lett. B 344 (1995) 259; Phys. Rev. D 52 (1995) 6224.

[11] S.W. Hawking, Phys. Rev. D 14 (1975) 2460; Commun. Math. Phys. 87 (1982) 395;

D.N. Page, Phys. Rev. Lett. 44 (1980) 301; Gen. Rel. Grav. 14 (1982) 299;

R.M. Wald, Phys. Rev. D 21 (1980) 2742;

J. Ellis, N.E. Mavromatos and D.V. Nanopoulos, CERN preprint TH.6755 (December 1992); (hep-th/9212057). 